\title{
Biologie De La Reproduction Du Sciaenidae Pseudotolithus Elongatus Dans La Lagune Ebrie (Cote d'Ivoire)
}

\section{Koné Tiéhoua \\ Kouakou Fokouo Kessia Irène}

(Laboratoire de biologie et de cytologies animales,)

UFR des sciences de la nature, Université Nangui Abrogoua, Côte d’Ivoire

\section{Konan Kouassi Sylvain}

(Département Aquaculture)

Centre de Recherches Océanologiques, Côte d’Ivoire

\section{N'Da Konan}

(Laboratoire de biologie et de cytologies animales,)

UFR des sciences de la nature, Université Nangui Abrogoua, Côte d'Ivoire

doi: 10.19044/esj.2016.v12n6p327 URL:http://dx.doi.org/10.19044/esj.2016.v12n6p327

\begin{abstract}
The study of reproduction of Pseudotolithus elongatus in the Ebrie lagoon was investigated between July 2013 and June 2015 on a whole of individuals of 550 specimens. the total length ranges from 14.5 to $42.7 \mathrm{~cm}$. The monthly evolution of the gonado-somatic index (GSI), the liver-somatic index (LSI), the condition factor (K), the Sex-ratio, the fecundity, the diameter of eggs revealed that this species spawns throughout the year but the main breeding period was in raining season. Sex-ratio observed was in favour of females (1: $\left.1.14 ; X^{2}=2.26 ; p>0.05\right)$. The percentage of mature specimens by sexes was calculated for each size class. Total length at first maturity was $21.06 \mathrm{~cm}$ for females and $20.21 \mathrm{~cm}$ for males. The ripe ovaries contained 39883-814757 eggs. The relative fecundity was 216-1979 eggs per $\mathrm{g}$ of body weight of females. And the mean diameter of eggs is $336 \pm 138$ microns.
\end{abstract}

Keywords: Pseudotolithus elongatus, reproduction, fecundity, Ebrie lagoon

Résumé

L'étude de la reproduction de Pseudotolithus elongatus de la lagune Ebrié a été faite entre juillet 2013 et juin 2015 sur un total de 550 spécimens (273 femelles, 239 mâles et 38 individus de sexe indéterminé) de longueur 
totale comprise entre 14,5 et 42,7 cm. Les paramètres de reproduction suivis mensuellement ont été le rapport gonado-somatique (RGS), le rapport hépato-somatique (RHS) le facteur de condition (K)le sex-ratio, la fécondité et le diamètre ovocytaire. La taille de première maturité sexuelle a été obtenue par le calcul du pourcentage des individus matures par classe de taille et par sexe.

Les résultats obtenus révèlent que cette espèce se reproduit toute l'année avec un fort taux de reproduction en saison pluvieuse. Le sex-ratio global montre en général une dominance des femelles par rapport aux mâles $\left(1: 1,14 ; x^{2}=2,26 ; p>0,05\right)$. La taille de première maturité sexuelle est obtenue par le calcul du pourcentage des individus matures par classe de taille et par sexe. Elle est de 21,06 cm et de 20,21 cm respectivement chez les femelles et les mâles.

La fécondité absolue varie de 39883 à 814757 ovocytes par ovaire avec une fécondité relative comprise entre 216 et 1979 ovocytes par gramme de poids corporel. Le diamètre moyen des ovocytes est de $336 \pm 138$ microns et fait ainsi de $P$. elongatus une espèce à petits œufs et à forte fécondité.

Mots clés : Pseudotolithus elongatus, reproduction, Fécondité,Lagune Ebrié

\section{Introduction}

Les scianidaes sont des poissons essentiellement marins, démersaux vivant sur les fonds sableux ou vaseux des régions côtières. Cette famille comprend plus de 200 espèces réparties en 50 genres environ. Sur les côtes ouest africaines, huit genres et 16 espèces sont reconnues. Parmi celles-ci seulement trois espèces sont rencontrées en eaux saumâtres dont Pseudotolithus elongatus avec une aire de distribution allant du Sénégal à l'Angola (Paugy et al., 1992). P. elongatus comme l'ensemble des poissons démersaux tient une place très importante dans le secteur des pêches, ceci notamment à cause de sa valeur commerciale extrêmement élevée, de sa présence régulière dans les débarquements et de la place de choix qu'il occupe dans l'assiette du consommateur (Sidibé 2010).

En Côte d'Ivoire, cette espèce est présente de façon permanente dans la zone estuarienne de la lagune Ebrié (Albaret, 1994) et fait alors partit des espèces régulièrement débarquées par la pêcherie artisanale commerciale. Cependant, très peu de données existent sur la biologie et l'écologie en général et sur la biologie de la reproduction en particulier. En effet La connaissance de la biologie de la reproduction des poissons est essentielle pour une bonne gestion des ressources halieutiques (Sylla, 2009).

La présente étude a pour objectifs de déterminer la période de reproduction et la fécondité de cette espèce dans nos eaux, sur la base de quelques paramètres tels que la taille de première maturité sexuelle, les 
indices gonado-somatiques et hépato-somatiques, le facteur de condition et les stades de maturation sexuelle.

\section{Zone d'étude, matériel et méthodes}

\section{Zone d'étude}

Située dans le sud de la Côte d'Ivoire, la lagune Ebrié est limitée à l'ouest par le canal d'Assagny et à l'est par le canal d'Assinie. C'est un système lagunaire (lagunes Adjin et Potou, baies de cocody et Bietry) de forme allongée avec une superficie de $566 \mathrm{~km}^{2}$ (Varlet, 1978). Située entre $3^{\circ} 47^{\prime}$ et $5^{\circ} 29^{\prime}$ ouest et $5^{\circ} 02^{\prime}$ et $5^{\circ} 42^{\prime}$ nord, la lagune a environ $130 \mathrm{~km}$ de longueur et une largeur maximum de $7 \mathrm{~km}$. Son volume total est d'environ $2,7.10^{9} \mathrm{~m}^{3}$. La profondeur moyenne de l'eau est de 4,8 m bien qu'on puisse observer des profondeurs de 20 m près d'Abidjan (Albaret, 1994 ; Durand et Guiral, 1994 ; Laë, 1997).

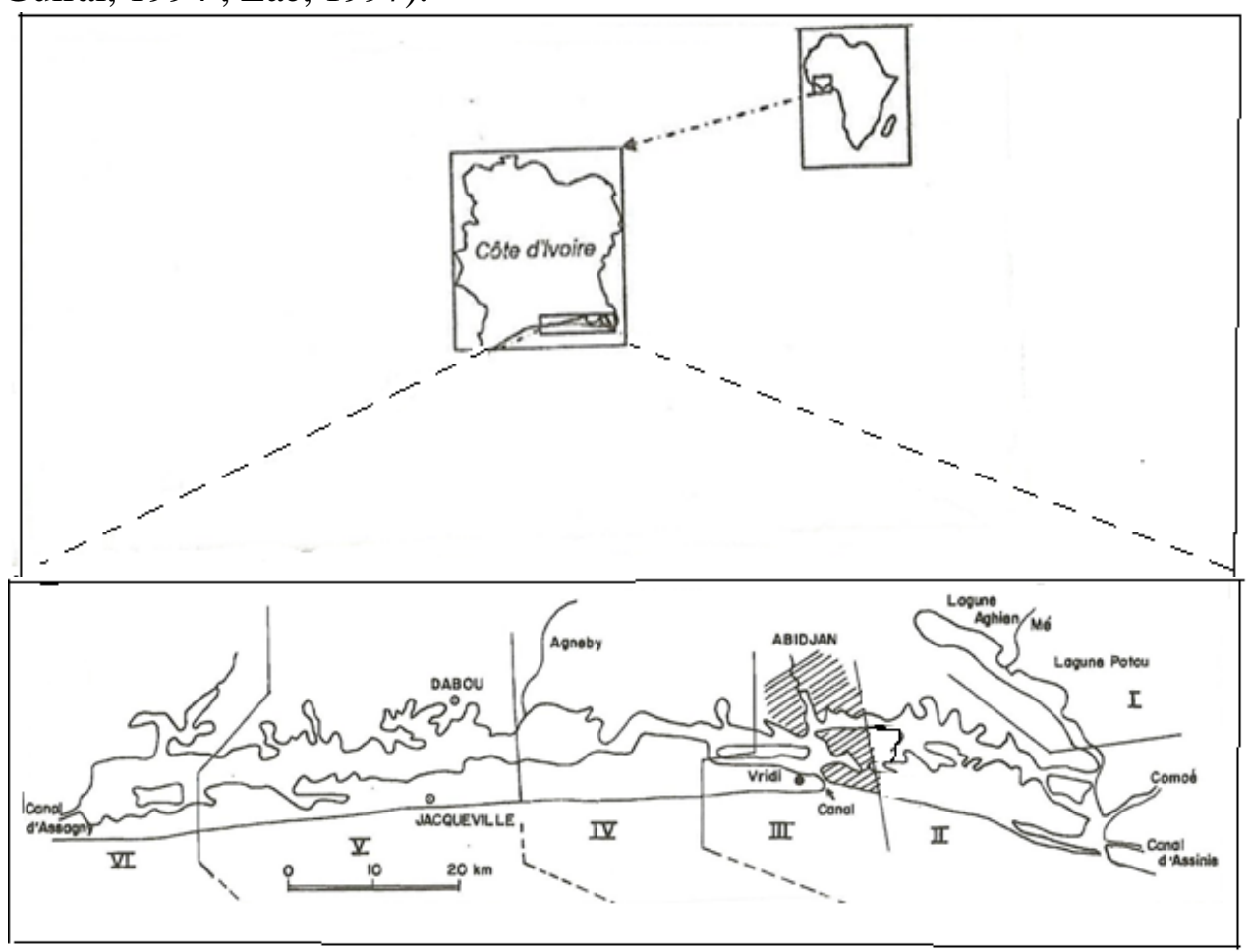

Figure 1 : Présentation de la zone d'étude

\section{Matériel et méthodes}

Les poissons ayant servi à cette étude ont été collectés mensuellement de juillet 2013 à juin 2015. Ceux-ci proviennent de la zone III de la lagune Ebrié. Un total de 550 spécimens capturés à l'aide de filets maillants (mailles: comprise entre 20 et $40 \mathrm{~mm}$ ) ont été identifié selon les clés de détermination de Paugy et al.(1992). 
Pour chaque spécimen, la longueur standard, la longueur totale ainsi que le poids vif et le poids éviscéré ont été respectivement relevés au millimètre et au gramme près. Après dissection le sexe a été déterminé, les gonades et les foies ont été prélevés et pesés au gramme près.

Les paramètres de reproduction que sont la taille de première maturité $\left(\mathrm{L}_{50}\right)$, Le sex-ratio (SR), le rapport gonado-somatique (RGS), le rapport hépato-somatique (RHS) et le facteur de condition (K) ont été déterminés ainsi que les fécondités absolue et relative.

\section{Taille de première maturité sexuelle}

L'échelle de maturité définie par Fontana (1969), Fontana et Le Guen (1969), a servi à la détermination des stades de maturités sexuelles. La détermination de la $\mathrm{L}_{50}$ (longueur à partir de laquelle $50 \%$ des poissons sont matures) a été faite en regroupant les individus échantillonnés par sexe et par classe de taille. Ensuite, la proportion des individus matures de chaque classe de taille a été déterminée. Les couples taille-proportion d'individus matures sont ajustés à une courbe logistique de type sigmoïde (Amenzoui, 2010) dont l'expression mathématique est la suivante :

$\mathrm{P}=1 /\left(1+\mathrm{e}^{-\left(\mathrm{a}+\mathrm{b}^{*} \mathrm{Lt}\right)}\right)$

$\mathrm{L}_{50}=-\frac{a}{b}$

$\mathrm{P}$ : Proportion des matures par classe de taille ; $\mathrm{L}$ : Longueur totale ; $\mathrm{a}$ et $\mathrm{b}=$ constantes

\section{Sexe ratio}

Le sex-ratio renseigne sur l'équilibre des sexes à l'intérieur d'une population.Il a été calculé à partir d'individus de sexe connu, car chez $P$. elongatus, les individus mâles et femelles ne peuvent être distingués morphologiquement. La dissection de la région abdominale est donc nécessaire pour déterminer le sexe.

$$
\mathrm{SR}=\frac{\mathrm{M}}{\mathrm{F}}
$$

SR : sex- ratio ; F : nombre de femelles ; M : nombre de mâles

\section{Rapport gonado-somatiques}

Le rapport gonado-somatiques (RGS) permet de déterminer les périodes de ponte au cours du cycle sexuel d'une espèce (Analbery, 2004).

$$
R G S=\frac{\text { Pg }}{\text { Pév }} \times 100
$$

Pg : poids des gonades en g ; Pév : poids éviscéré en g

Une moyenne mensuelle des RGS est calculée à partir des données individuelles. 


\section{Rapport hépato-somatique (RHS)}

Chez les poissons, l'ovogenèse et la spermatogenèse nécessitent un apport important d'énergie qu'ils stockent dans le foie essentiellement sous forme de lipides. Les variations pondérales hépatiques sont influencées par les conditions génitales des poissons (Konan, 2013).

$$
R H S=\frac{\text { Pf }}{\text { Pév }} \times 100
$$

Pf : poids du foie en g ; Pév : poids éviscéré en g

\section{Facteur de condition}

Le facteur de condition (K) permet de traduire l'embonpoint du poisson par rapport à l'effet des facteurs écologiques et physiologiques.Ce coefficient nous renseigne sur le stockage des réserves nécessaires à la gamétogenèse (Fehri-bedoui, 2002).

$K=\frac{P}{\mathrm{LT}^{3}} \times 100$

\section{Fécondités}

Les fécondités absolue (nombre d'ovocytes dans un ovaire) et relative (nombre d'ovocyte par unité de poids corporel) ont été évaluées pour déterminer le potentiel reproducteur d'une espèce. La fécondité a été déterminée à partir de comptages des ovocytes dans un sous-échantillon d'ovaire de poids connu et constitué de trois morceaux prélevés dans les régions rostrale, moyenne et caudale (Montchowui et al., 2007). Seuls les ovaires des femelles au stade IV et V ont été pris en compte. Le nombre total d'ovocytes dans l'ovaire (N) et la fécondité relative (Fr) sont calculés par les formules suivantes:

$$
N=\frac{\mathrm{n} \times \mathrm{Pg}}{\mathrm{p}} F r=\frac{\mathrm{N}}{\mathrm{Pp}}
$$

$\mathrm{N}$ : nombre total d'ovocytes dans l'ovaire ou fécondité absolue ; n : le nombre d'ovocytes dans l'échantillon ; Pg : poids de l'ovaire (g) ; P : poids de l'échantillon (g) ; Pp : poids plein du poisson (g).

Le diamètre des ovocytes a été mesuré à l'aide d'un micromètre.

\section{Resultats}

\section{Sex-ratio et taille de première maturité sexuelle.}

Sur un ensemble de 550 spécimens de $P$. elongatus échantillonnés, 273 femelles et 239 mâles ont été identifiés. Les individus de sexe non déterminé étaient au nombre de 38. Les femelles sont numériquement plus nombreuses que les mâles. Le sex-ratio (Mâles: Femelle) associés à ces valeurs est de 1:1,14 c'est-à-dire 1,14 femelles pour 1 mâle (Tableau 1). Ce sex-ratio n'est pas significativement différent du sex-ratio théorique $1: 1\left(x^{2}=2,26 ; p\right.$ 
$>0,05)$. La variation mensuelle du sex-ratio montre qu'il n’y a pas de différence significative avec la valeur théorique 1:1 tout au long de notre étude excepté les mois de février et août 2014 où la différence est significative $\left(\mathrm{x}^{2}=7,54\right.$ et $\left.4 ; \mathrm{p}<0,05\right)$.

La comparaison des données de sex-ratio entre les saisons pluvieuses et les saisons sèches n'indique pas de différence significative ( $x^{2}=0,21$ et 2,58; $\mathrm{p}>0,05$ ) bien que le nombre de femelles soit plus élevé que le nombre de mâles.

Les tailles de première maturité sexuelle sont de 21,06 cm chez les femelles (figure 2) et de 20,21 cm chez les mâles (figure 3). Les mâles atteignent leur maturité sexuelle à une taille inferieure à celle des femelles. La différence de taille n'est pas statistiquement significative $\left(\mathrm{x}^{2}=0,02 ; \mathrm{p}>\right.$ 0,05).Le plus petit individu mature obtenu est de $18 \mathrm{~cm}$ pour les femelles et de 17,5 cm pour les mâles.

\begin{tabular}{|c|c|c|c|c|c|}
\hline Mois & $\begin{array}{l}\text { Effectifs } \\
\text { femelles }\end{array}$ & $\begin{array}{c}\text { Effectifs } \\
\text { mâles }\end{array}$ & $\begin{array}{c}\text { Effectifs } \\
\text { total }\end{array}$ & $\begin{array}{c}\text { sex-ratio } \\
\text { (M: F) }\end{array}$ & $\chi^{2}$ \\
\hline Juillet 2013 & 9 & 12 & 21 & $1: 0,75$ & 0,43 \\
\hline août & 11 & 11 & 22 & $1: 1$ & 0.00 \\
\hline septembre & 15 & 12 & 27 & $1: 1,25$ & 0,33 \\
\hline octobre & 14 & 7 & 21 & $1: 2$ & 2,33 \\
\hline novembre & 8 & 9 & 17 & $1: 0,88$ & 0,06 \\
\hline décembre & 19 & 13 & 32 & $1: 1,46$ & 1,13 \\
\hline janvier & 12 & 8 & 20 & $1: 1,5$ & 0,8 \\
\hline février & 20 & 6 & 26 & $1: 3,33$ & $7,54^{*}$ \\
\hline mars & 16 & 7 & 23 & $1: 2,28$ & 3,52 \\
\hline avril & 8 & 9 & 17 & $1: 0,88$ & 0,06 \\
\hline mai & 8 & 10 & 18 & $1: 0,8$ & 0,22 \\
\hline juin 2014 & 12 & 11 & 23 & $1: 1,09$ & 0,04 \\
\hline juillet & 9 & 11 & 20 & $1: 0,81$ & 0,2 \\
\hline août & 4 & 12 & 16 & $1: 0,33$ & $4^{*}$ \\
\hline Septembre & 16 & 15 & 31 & $1: 1,06$ & 0,03 \\
\hline octobre & 13 & 6 & 19 & $1: 2,16$ & 2,58 \\
\hline novembre & 9 & 8 & 17 & $1: 1,12$ & 0,06 \\
\hline décembre & 8 & 13 & 21 & $1: 0,61$ & 1,19 \\
\hline janvier & 10 & 8 & 18 & $1: 1,25$ & 0,22 \\
\hline février & 8 & 11 & 19 & $1: 0,72$ & 0,47 \\
\hline mars & 16 & 12 & 28 & $1: 1,33$ & 0,57 \\
\hline avril & 8 & 8 & 16 & $1: 1$ & 0 \\
\hline mai & 8 & 11 & 19 & $1: 0,72$ & 0.47 \\
\hline juin 2015 & 12 & 9 & 21 & $1: 1,33$ & 0,43 \\
\hline TOTAL & 273 & 239 & 512 & $1: \mathbf{1 , 1 4}$ & 2,26 \\
\hline saisons pluvieuses & 118 & 111 & 229 & $1: 1,06$ & 0,21 \\
\hline saisons sèches & 155 & 128 & 283 & $1: 1,21$ & 2,58 \\
\hline
\end{tabular}

Tableau 1 : variation mensuelle et saisonnière du sex-ratio de Pseudotolithus elongatus de juillet 2013 à juin 2015.* = différence significative 


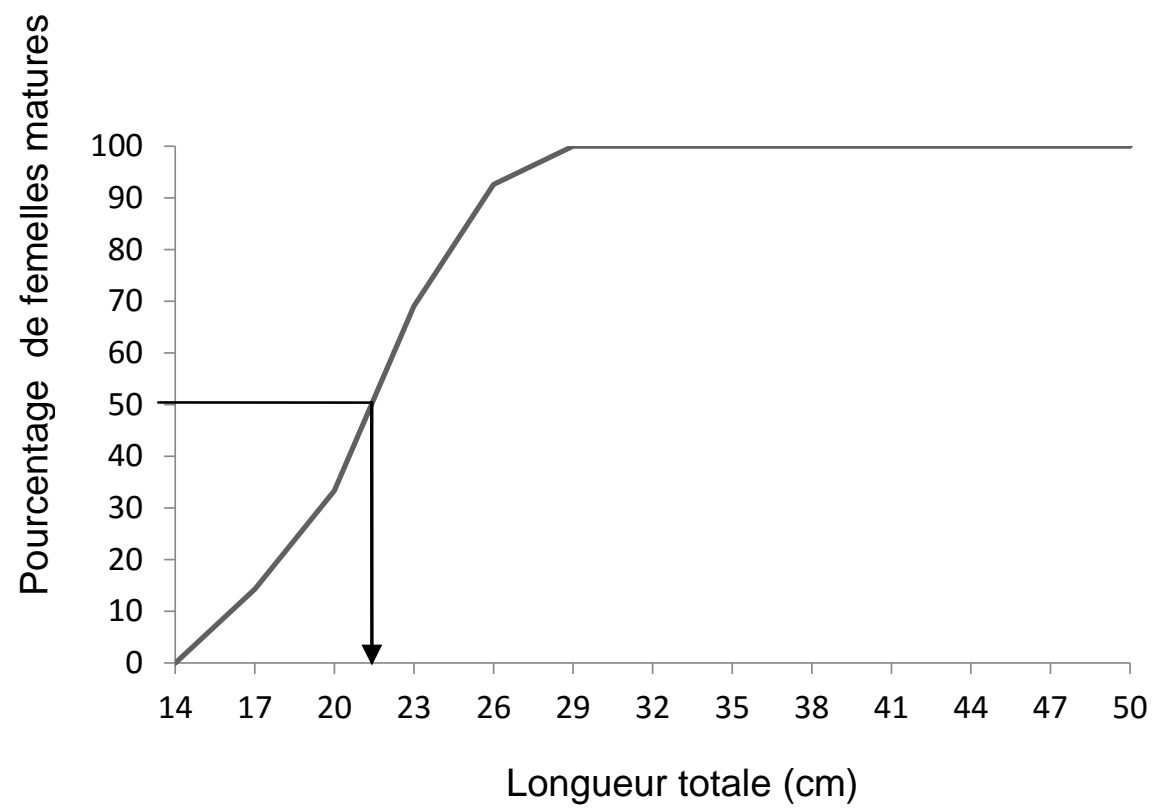

Figure 2 : Evolution de la maturite sexuelle en tonction de la taille (Lt) chez les femelles de P. elongatus provenant de la lagune Ebrié.

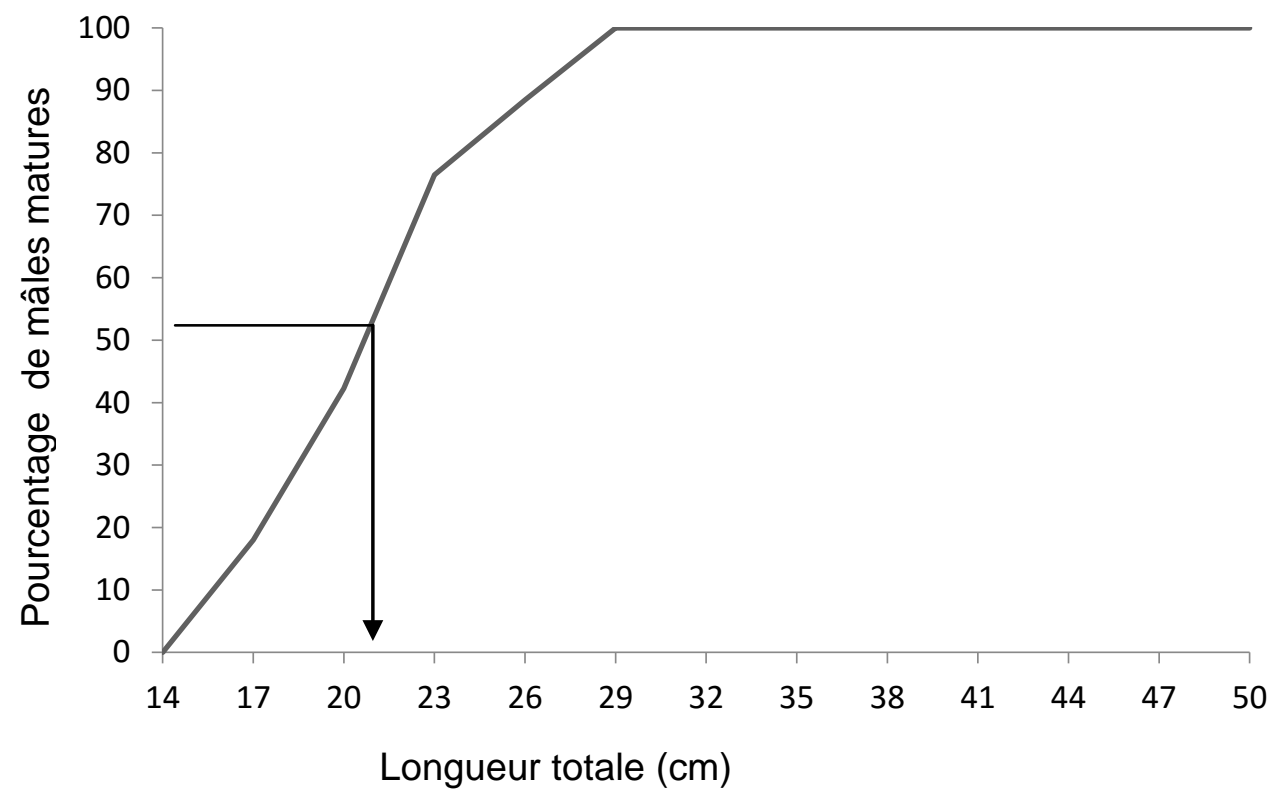

Figure 3 : Evolution de la maturité sexuelle en fonction de la taille (Lt) chez les mâles de $P$. elongatus provenant de la lagune Ebrié. 


\section{Variation mensuelle des stades de maturité sexuelle}

Les variations mensuelles des différents stades de maturités sexuelles révèlent que des femelles à différents stades de maturité sexuelle sont rencontrées sur toute l'année mais avec des proportions variables. Les femelles de stades de maturation sexuelle I sont présentes dans les échantillons durant les mois de septembre, janvier, mars et juin en ce qui concerne la première année, puis en septembre et juin l'année suivante. Le stade II est observé en août, septembre, décembre, janvier, février, mars et juin lors de la première année. Quant à la deuxième année le stade II est présent en septembre, janvier, mars et juin. Le stade III est observé sur toute la période d'échantillonnage excepté le mois d'avril 2014 et le mois de décembre 2015. Le maximum est atteint au mois de juin. Toutefois, les individus au stade de préponte et de ponte (stade IV et V) sont présents tous les mois de l'année. Un maximum d'individus au stade IV est observé en janvier en ce qui concerne la première année et en février de la deuxième année. Le maximum de spécimens au stade $\mathrm{V}$ est présent au mois d'avril 2014 et avril 2015. Quant au stade de post-ponte (stade VI), il est quasiment présent toute l'année excepté les mois d'octobre, décembre et août 2014. Le maximum est atteint en novembre 2013 et en novembre 2014.

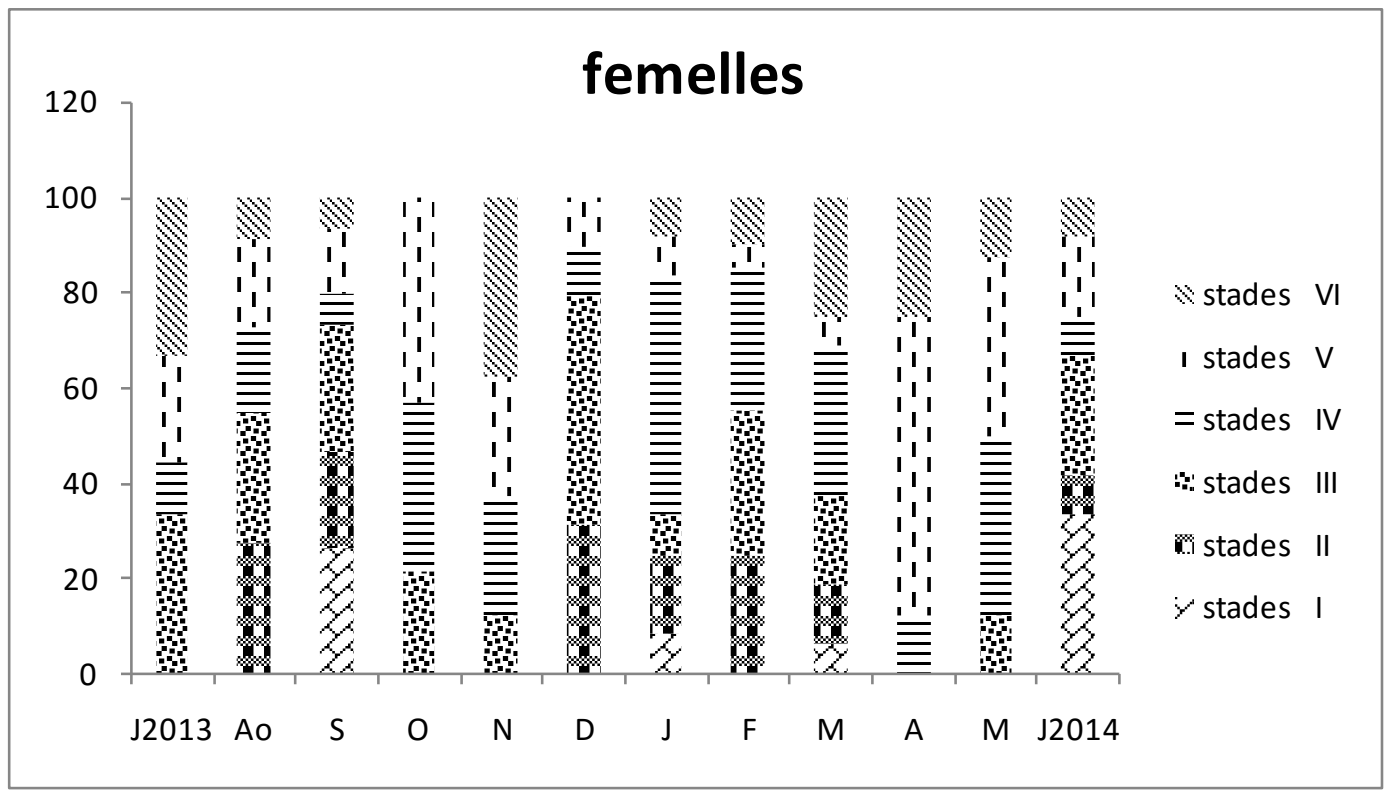

Figure 4 : Evolution des stades de maturité sexuelle des femelles de $P$. elongatus de la lagune Ebrié de 2013 à 2014. 


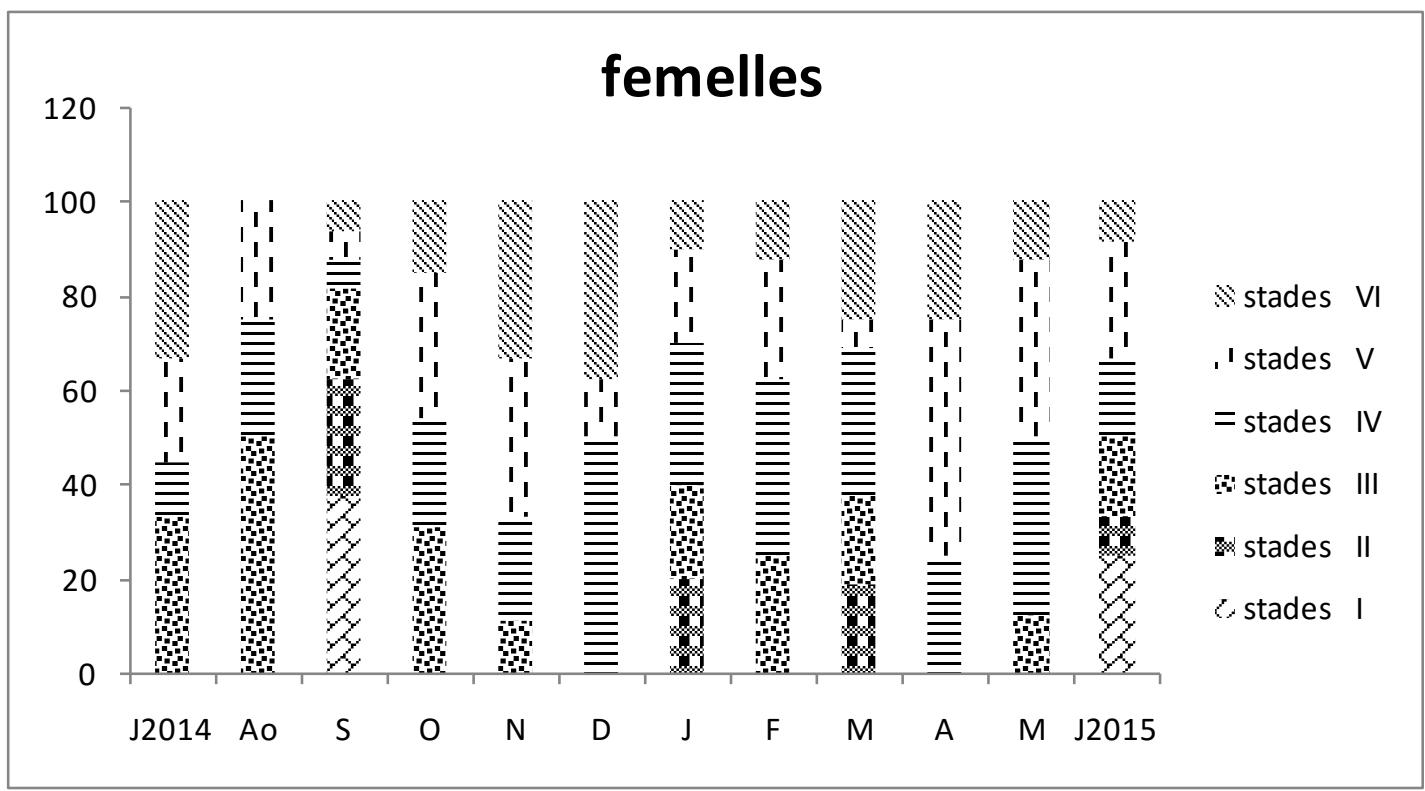

Figure 5 : Evolution des stades de maturité sexuelle des femelles de P. elongatus de la lagune Ebrié de 2014 à 2015.

\section{Variation mensuelle du rapport gonado-somatique (RGS) et période de reproduction}

L'évolution mensuelle du RGS pendant les deux cycles consécutifs de reproduction montre que les courbes du RGS suivent une évolution similaire d'une année à l'autre (figures 6 et 7). Dans l'ensemble, le RGS des femelles est plus élevé que celui des mâles.

Les courbes de variations mensuelles du RGS chez les femelles comme chez les mâles évoluent en dents de scie et font ressortir plusieurs pics.

Chez les femelles les valeurs les plus importantes du RGS sont observées en avril, juillet et octobre pour les deux années. Pour la première année d'étude les différentes valeurs des RGS sont: juillet 2013 (3,96 \pm 1,65), octobre $2013(4,2 \pm 2,96)$ et avril $2014(4,75 \pm 2,34)$.

En ce qui concerne la deuxième année les valeurs de juillet 2014, octobre 2014 et avril 2015 sont respectivement $(4,67 \pm 1,57)$, (3,18 $\pm 1,3)$ et $(4,38 \pm 2,29)$. Les faibles valeurs sont enregistrées en février 2014 (2,44 \pm $0,79)$ et décembre $2014(1,85 \pm 0,16)$. Chez les mâles le RGS atteint son niveau maximal en mai avec une valeur de 1,81 $\pm 0,14$ la première année et 2,04 $\pm 0,58$ la deuxième année.Les autres pics sont de moindre importance et s'observent en juillet $2013(1,54 \pm 0,46)$ décembre $2013(1,25 \pm 0,49)$ et mars2014 $(1,44 \pm 0,1)$. Les pics de faibles valeurs sont mentionnés en août $2014(1,57 \pm 0,46)$, novembre $2014(1,14 \pm 0,19)$ et février2015 $(1,67 \pm$ $0,55)$. Ces variations mensuelles révèlent que cette espèce se reproduit toute 
l'année avec une intensité plus importante en grande saison pluvieuse c'està-dire d'avril à juillet.
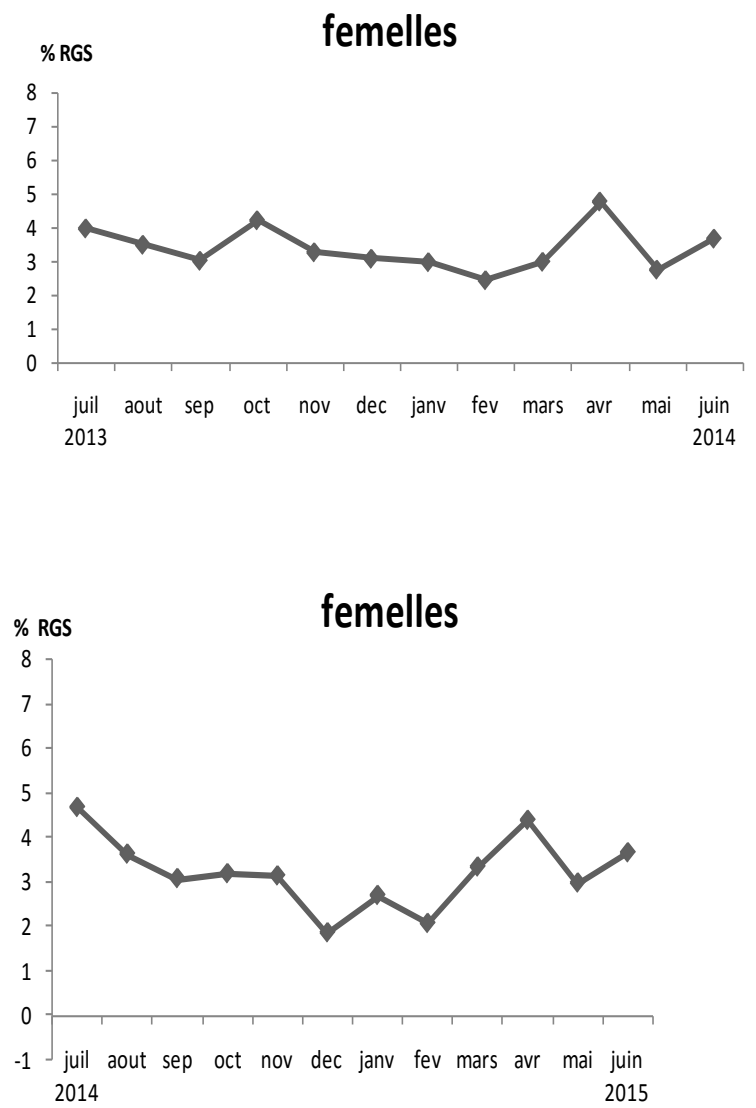

Figure 6 : Variation mensuelle du rapport gonado-somatique (RGS) des femelles de $P$. elongatus capturés dans la lagune Ebrié de 2013 à 2015.

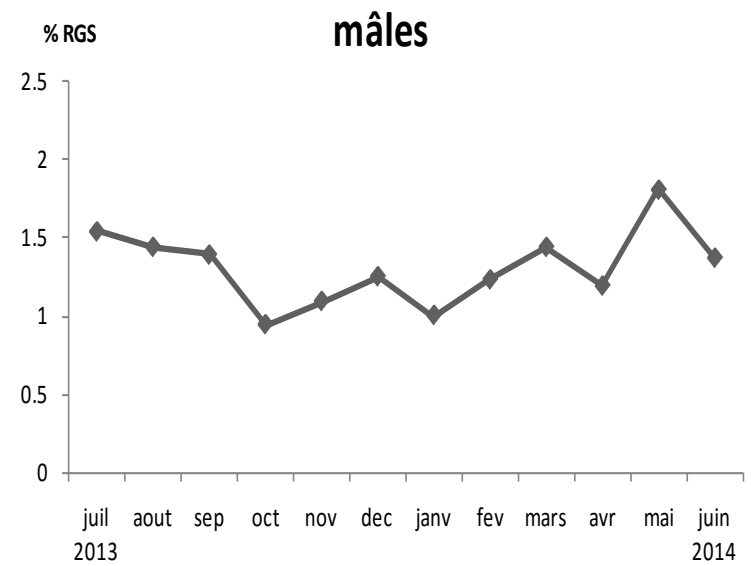




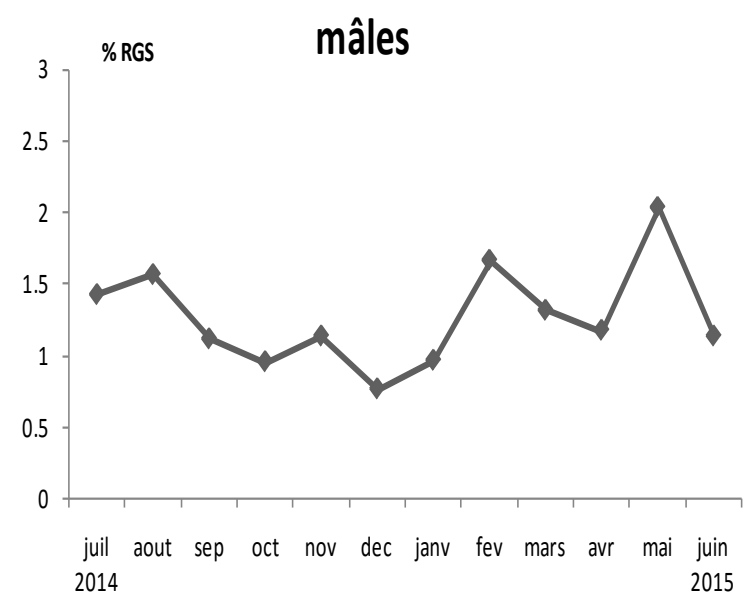

Figure 7 : Variation mensuelle du rapport gonado-somatique (RGS) des mâles de P. elongatus capturés dans la lagune Ebrié de 2013 à 2015.

Variation mensuelle du rapport hépato-somatique (RHS) et du facteur de condition (K) Variation mensuelle du rapport hépato-somatique (RHS)

L'évolution mensuelle du RHS chez les deux sexes (Figures 8 et 9) est similaire à celle du RGS durant les deux années consécutives. Les courbes des RHS évoluent en dent de scie et dans le même sens que celles des RGS. Chez les femelles le RHS atteint sa valeur maximale au mois d'avril en ce qui concerne les deux années. Le RHS moyen du mois d'avril est 2,24 $\pm 0,71$ pour la première année et $2,04 \pm 0,18$ pour la deuxième année. Chez les mâles le RHS moyen mensuelle le plus élevé est observé au mois de mai. Les valeurs sont de 1,81 $\pm 0,14$ et 1,54 $\pm 0,14$ respectivement en 2014 et 2015.

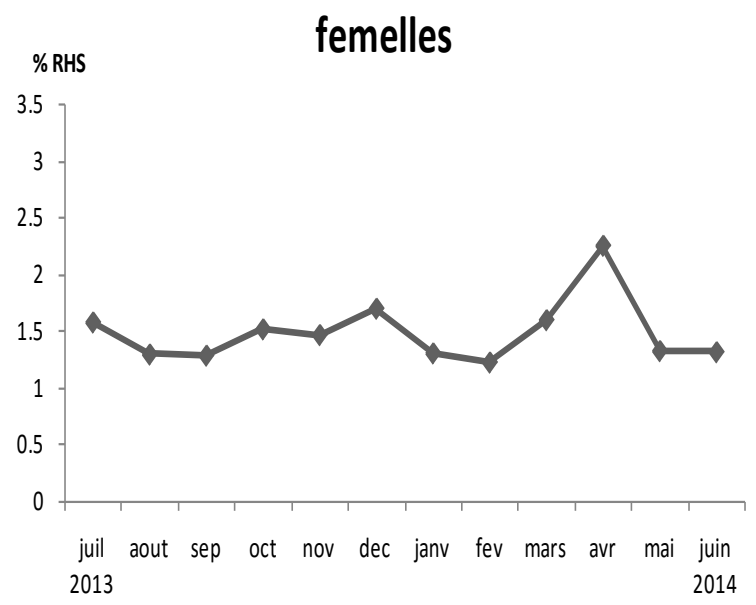




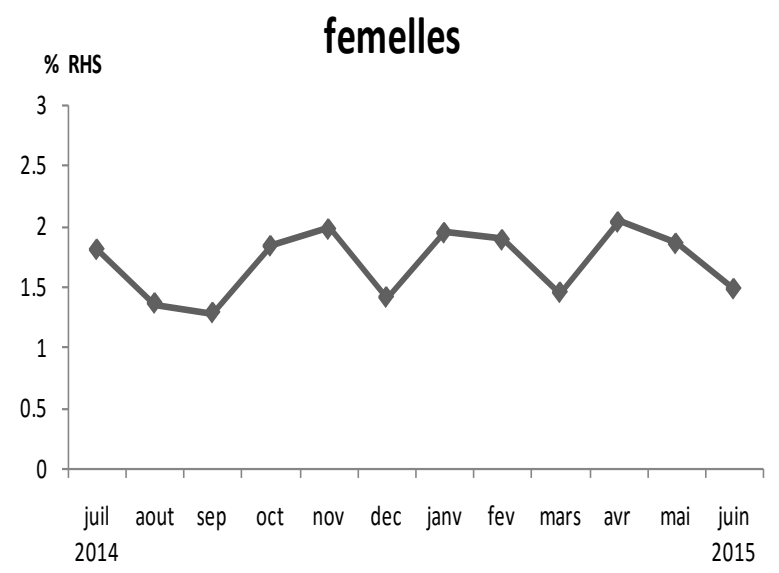

Figure 8 : Variation mensuelle du rapport hépato-somatique (RHS) des femelles de $P$. elongatus capturés dans lagune Ebrié de 2013 à 2015.
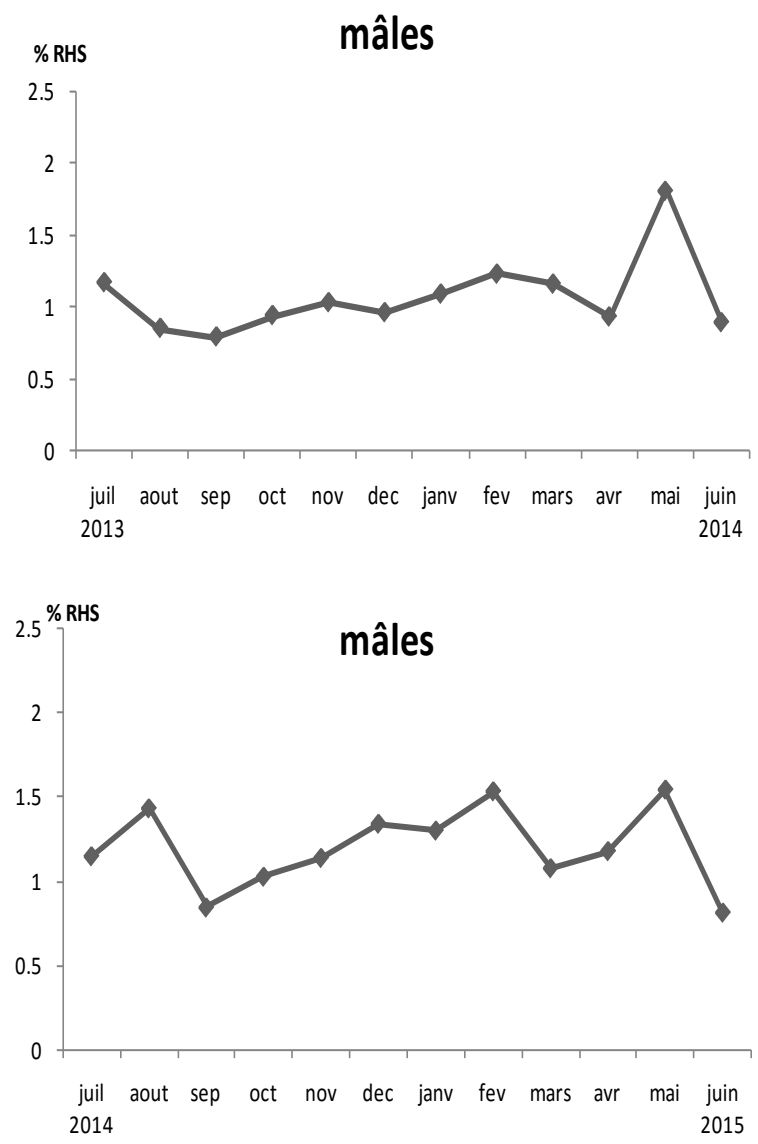

Figure 9 : Variation mensuelle du rapport hépato-somatique (RHS) des mâles de P. elongatus capturés dans la lagune Ebrié de 2013 à 2015. 


\section{Variation mensuelle du facteur de condition (K)}

Les figures 10 et 11 traduisent la variation du facteur de condition des individus femelles et mâles. Les valeurs maximales du facteur de condition $(\mathrm{K})$ chez les femelles sont observées aux mois de février $(0,99 \pm$ 0,06 et $0,96 \pm 0,12$ respectivement en 2014 et 2015) et les valeurs minimales sont enregistrées aux mois de juin $(0,8 \pm 0,01)$ pour la première année et en septembre $(0,8 \pm 0,03)$ en ce qui concerne la deuxième année. Chez les mâles K est plus élevés en juin $2014(0,85 \pm 0,02)$ et en décembre $2014(0,91 \pm 0,05)$ Les valeurs les plus faibles au cours des deux années s'observent pendant le mois de décembre $(0,76 \pm 0,04)$ durant la première année et en avril $(0,70 \pm$ $0,02)$ en ce qui concerne la deuxième année.
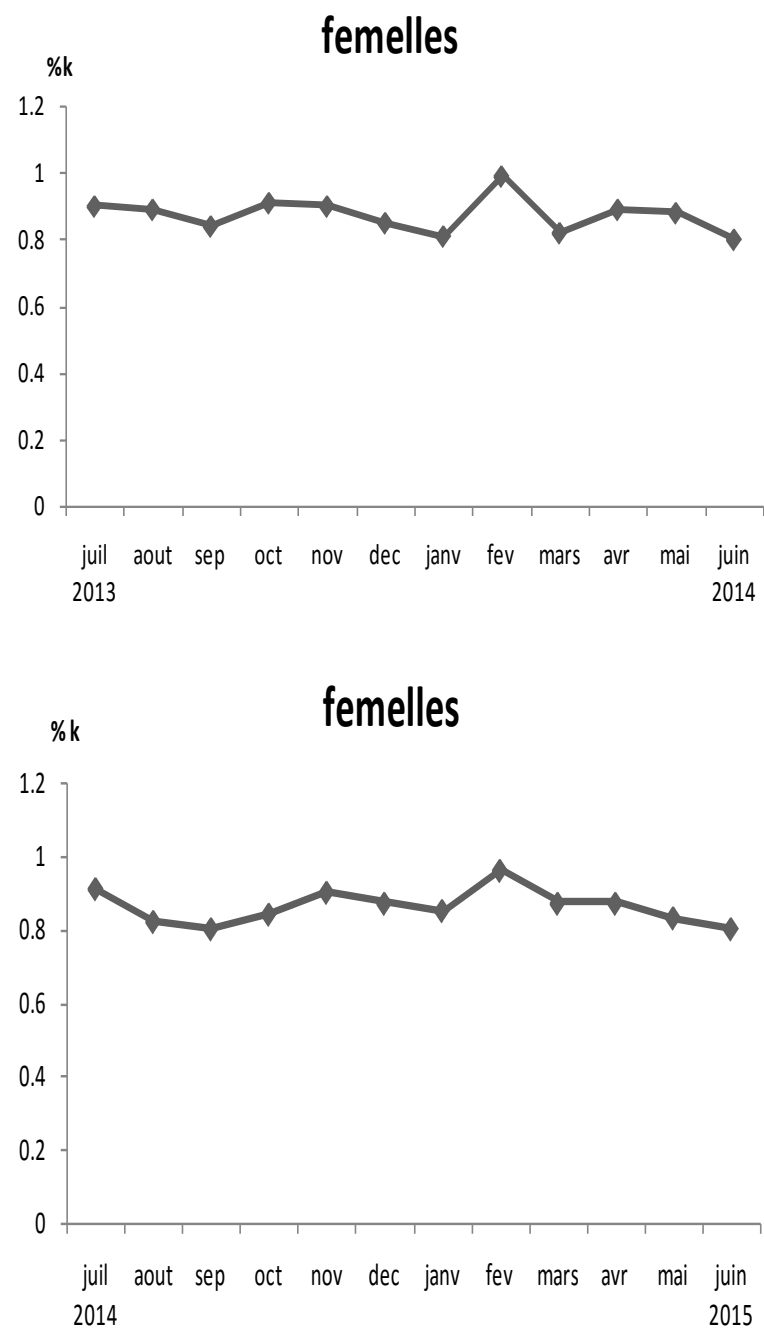

Figure 10 : Variation mensuelle du facteur de condition (K) des femelles de P. elongatus capturés dans la lagune Ebrié de 2013 à 2015. 

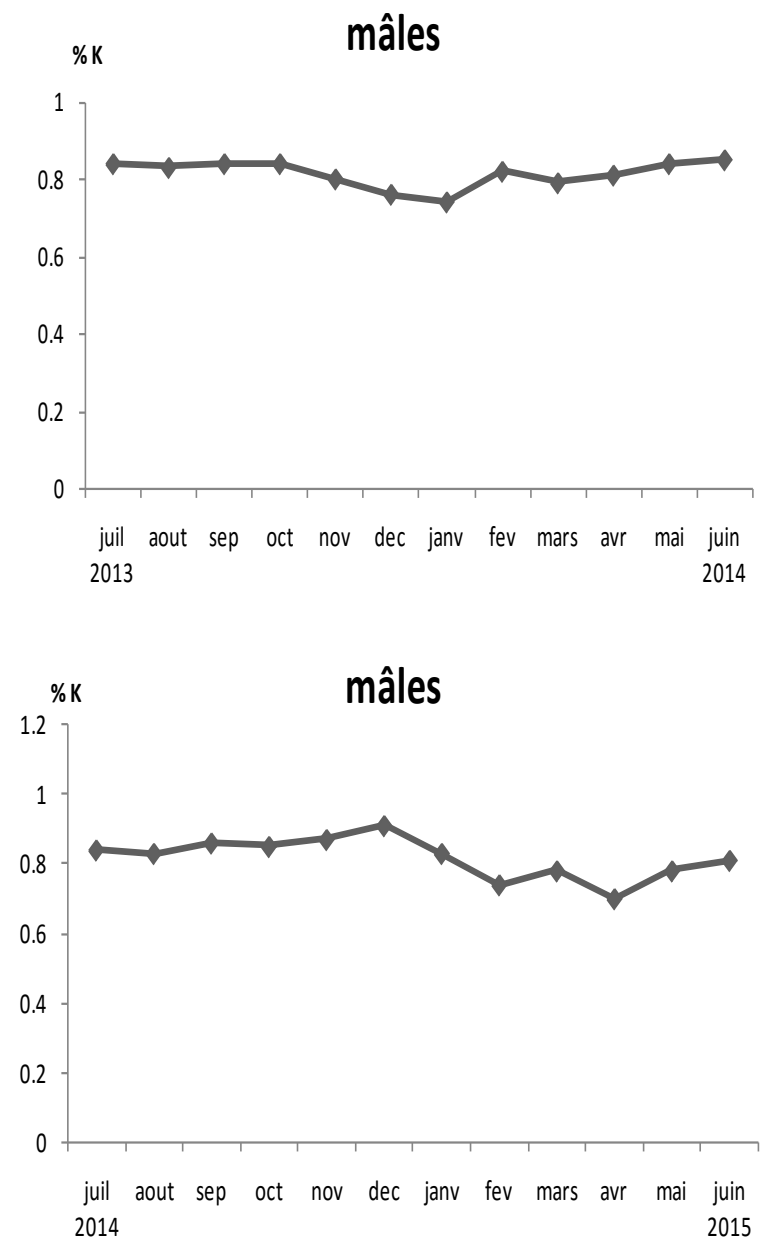

Figure 11 : Variation mensuelle du facteur de condition (K) des mâles de P. elongatus capturés dans la lagune Ebrié de 2013 à 2015.

\section{Fécondité}

Le nombre d'ovocytes par ovaire (fécondité absolue) varie de 39883 à 814757 ovocytes avec une valeur moyenne de $184602 \pm 172802$ ovocytes. Les spécimens ont une longueur totale comprise entre 23,5 et 42,7 cm et un poids variant entre 115 et $807 \mathrm{~g}$. Quant à la fécondité relative, elle est comprise entre 216 et 1.979 ovocytes par gramme de poids corporel, avec une valeur moyenne de $736 \pm 437$ ovocytes. Les ovocytes ont un diamètre compris entre 192 et 810 microns. Le diamètre moyen des ovocytes est de $336 \pm 138$ microns. 


\section{Discussion}

Le sex-ratio 1:1,14 observé chezl'ensemble de la population de Pseudotolithus elongatusmontre qu'il y a une dominance des femelles par rapport aux mâles. La variation saisonnière révèle laprédominance des femelles en toutes saisons. Cette prédominance de l'un des sexes est un phénomène relativement fréquent chez de nombreuses espèces de poissons téléostéens (Djadji et al., 2013 ; Poulet, 2004). Selon Sylla (2009), plusieurs facteurs tels que le déplacement pour la recherche de nourriture, la croissance différentielle et le taux de mortalité par sexe influencent également le sex-ratio chez les poissons.

D’après Caverivière (1982), cette dominance des femelles chez les espèces de la communauté des Sciaenidae pourrait aussi s'expliquer par une disponibilité ou une capturabilité plus grande des femelles, une mortalité naturelle plus élevée chez les mâles, ou plus simplement une croissance différentielle.

Les résultats obtenus sont similaires à ceux de Olapade (2014) et Sossoukpe et al., (2013) respectivement sur Pseudotolithus senegalensis en Sierra Leone et sur Pseudotolithus typus au Benin. Koné et al., (2011) lors des travaux réalisés sur Pellonula leonensis dans le lac de barrage de Kossou en Côte d' Ivoire ont également remarqué que l'ensemble des captures de $P$. leonensis était dominé par des femelles. Ameur et al., (2003) et Mohamed (2004) ont signalé une dominance des femelles chez les mulets respectivement à l'embouchure de la lagune Merja zerga avec l'Océan Atlantique et sur les côtes mauritaniennes.

La taille de première maturité sexuelle $\left(\mathrm{L}_{50}\right)$ de Pseudotolithus elongatusest atteinte à la taille (longueur totale) de 20,21 cm chez les mâles et $21,06 \mathrm{~cm}$ chez les femelles.

Selon Koné et al. (2011) les différences de tailles observées s'expliquent par le fait que la taille de maturité des individus dépend des facteurs biologiques et/ou écologiques du milieu, des activités anthropiques telles que la pêche abusive, et la répartition de l'énergie pour la production des gamètes.Les résultats obtenus indiquent que les mâles atteignent leur maturité sexuelle avant les femelles. Des résultats semblables ont été observés chez Trachinotus teraia de la lagune Ebrié par Sylla (2009). Cependant ceux obtenus par Koné et al. (2011) sur Pellonullaleonensis dans le lac Kossou sont différents selon ces auteurs, les individus femelles atteignent leur maturité sexuelle avant les mâles. Par ailleurs, d'autres résultats différents ont été également observés par Domain et al. (2000) en Guinée $\left(\mathrm{L}_{50}=24,5 \mathrm{~cm}\right)$; N'Jock (1990) au Cameroun $\left(\mathrm{L}_{50}=19,5\right) \mathrm{cm}$; Fontana et Le Guen (1969) au Congo $\left(\mathrm{L}_{50}=30 \mathrm{~cm}\right)$ lors de leurs travaux réalisés sur le $P$. elongatus. La variabilité de la taille de première maturité sexuelle d'une zone biogéographique à une autre pourrait être fonction des 
conditions physicochimique du milieu, de la disponibilité en nourritures. Poulet (2004) affirme que cette différence peut se justifier par la capacité d'adaptation des reproducteurs aux facteurs environnementaux à savoir la température, la salinité et ressources trophiques.

L’analyse combinée des RGS et des stades de maturité sexuelle a montré que $P$. elongatus se reproduit sur toute l'année avec une période de reproduction maximale entre avril et juillet. Ces résultats confirment ceux de Enakem et al (2004) réalisés sur la même espèce dans la rivière Cross au Nigeria. Ces auteurs ont remarqué que cette espèce se reproduit le long de l'année avec une reproduction intense de décembre à février. Ces résultats sont également en accord avec ceux de Domain et al. (2000) réalisés sur $P$. elongatus en Guinée. Selon ces auteurs la reproduction de cette espèce a lieu toute l'année.

La comparaison des courbes de variations mensuelles du RGS et du RHS permet de constater que les deux rapports évoluent de façon synchrone. Le maximum du RGS coïncide avec celui du RHS. L'augmentation du poids du foie apparaît bien liée à l'accroissement de l'activité génitale. Ce résultat s'explique par le fait que $P$. elongatusest un poisson gras. Il stocke les réserves d’énergie essentiellement dans les muscles, dans les mésentères périviscéraux et sous la peau. Ces réserves passent ensuite dans le foie et dans les gonades pour assurer les besoins énergétiques en période de reproduction d'où la faible valeur du facteur de condition pendant la période de reproduction (Djadji et al 2013). Ces résultats s'accordent avec ceux de Olapade (2014) réalisé sur Pseudotolithus senegalensis en Sierra Léone et ceux de Enakem et al (2004) réalisés sur $P$. elongatus dans la rivière Cross au Nigeria.

La fécondité absolue varie de 39883 à 814757 ovocytes et la fécondité relative est comprise entre 216 et 1979 ovocytes par gramme de poids corporel. Ce résultat révèle que cette espèce est un poisson à forte fécondité. Au Nigerialors de leur études réalisés sur $P$. elongatusEnakem et al. (2004) ont obtenu des résultats semblables aux nôtres (fécondité relative moyenne $=1319$ ovocytes par gramme de poids corporel).Ces résultats sont également similaires à ceux relevés par Fontana et Le Guen (1969) sur la même espèce lors des études réalisée au Congo (fécondité absolue variant entre 14000 et 420000 ovocytes). Sossoukpe et al.(2013) ont obtenus un résultat similaire (fécondité relative moyenne $=63655 \pm 1.080$ ) en réalisant la même étude sur une espèce du même genre au Benin (Pseudotolithus typus). Les fortes valeurs de fécondité observées pourraient situer $P$. elongatus au rang des espèces très prolifiques qui produisent de nombreux œufs de petites tailles. Cela permet à cette espèce de vite reconstituer son stock. Cette forte fécondité peut être une stratégie mise en place par les 
espèces qui ne pratiquent pas la protection parentale afin de maximiser le taux de survivants de leurs progénitures (Sylla 2009).

\section{Conclusion}

La reproduction de Pseudotolithuselongatus a lieu toute l'année avec une période plus favorable en saison pluvieuse. Ainsi, la ponte de $P$. elongatus est maximale en avril, juillet et octobre.La première maturité sexuelle est atteinte à la taille de 21,06 cm de longueur totale chez les femelles et de 20,21 cm de longueur totale chez les mâles. Les fécondités observées montrent que $P$. elongatus appartient à la catégorie des espèces à petits œufs et à forte fécondité.

\section{References:}

Albaret J.J. Les poissons: Biologie et peuplement. InEnvironnement et ressources aquatiques de Côte d'Ivoire. Tome II- Les milieux lagunaires (Durand J. R., Dufour, P. Guiral, D. and Zabi, S. G. Eds.). ORSTOM, Paris : 239-274. 1994.

Amenzoui K. Variabilité des caractéristiques biologiques de la sardine, Sardinapilchardus (Walbaum, 1792) exploitée au niveau des zones de Safi, Agadir et Laâyoune (côtes atlantiques marocaines). Thèse de doctorat de l’université Mohammed v - Agdal 329p. 2010.

Ameur B., Bayed A., Benazzou T. Rôle de la communication de la lagune de Merja Zerba (Gharb, Maroc) avec l’océan Atlantique dans la reproduction d'une population de Mugil cephalus L. (Poisson Mugilidae). Bulletin de l'Institut Scientifique, Rabat, sectionSciences de la Vie, 25 : 77-82. 2003.

Analbery M. Biologie, écologie et pêche de Hemiramphus brasiliensis. Thèse de doctorat de l'université de Bretagne 61-123. 2004.

Caveriviere A. Les espèces demersales du plateau continental ivoirien ; biologie et exploitation. Thèse a l'université d'Aix- Marseille Faculté dès Sciences de Luminy. 1 : 159p.1982.

Djadji G. L., Atse B. C., Sylla S., Konan K. J., Kouassi N. J. Reproduction du Mugilidae Mugil cephalus Linné, 1758 dans deux complexes lagunaires (lagunes Ébrié et de Grand-Lahou) de la Côte d'Ivoire.International Journal of Biologie and Chemical Sciences 7(4): 1701-1716. 2013.

Domain F., Chavance P., Bah A. Notes sur la reproduction des espèces démersales du peuplement côtier guinéen. In La Pêche Côtière en Guinée Ressources et Exploitation. (Domain F., Chavance P., Diallo A. eds.). CNSHB,393 p. 2000.

Durand J. R., Guiral D. Hydroclimat et hydrochimie.59-90. InEnvironnement et ressources aquatiques de Côte d'Ivoire,d'Ivoire. Tome II - Les milieux lagunaires, (Durand J. R., Dufour P.,Guiral D., Zabi S. G., Eds.).ORSTOM, Paris, 543p. 1994. 
Enakem S. B., Achima M. J., Ekere N. M. Studies of some reproductive of Pseudotolithus elongatus in the Cross River estuary,Nigeria.Scientia. Marina 68(2): 265-271.2004.

Fehri-bedoui, R., Gharbi H., Abed A. Période de reproduction et maturité sexuelle de Liza aurata (poisson Mugilidae) des côtes Est et Sud tunisiennes. Bulletin de l'Institut National des Sciences et Technique Mer, 29: 1115.2002.

Fontana A., Le Guen J. C. Étude de la maturité sexuelle et de la fécondité de Pseudotolithus (fonticulus) elongatus. ORSTOM, série Océanographique, 7(3) : 9-19. 1969.

Fontana A.

Etude de la maturité sexuelle des sardinelles Sardinella ebo et Sardinelle aurita. de la région de Pointe Noire. Cahiers ORSTOM, série Océanographie, 7(2) : 101-114. 1969.

Konan. K. S. Biologie et pêche des poissons capitaines : polydactylus quadrafilis, Galeoides decadactilus et pentanemus quinqarius de la pêcherie artisanale maritime de Grand-Lahou,Côte d'Ivoire. Thèse de doctorat de l’Université Nangui Abrogoua , 184p. 2013.

Koné N., Berte S., Kraidy A. L. B., Kouamelan E. P., Koné T. Biologie de la reproduction du Clupeidae Pellonula leonensis (Boulenger, 1916) dans le lac de barrage de Kossou (Côte d'Ivoire). Journal of Applied Biosciences 41: 2797 - 2807. 2011.

Laë R. Does overfishing lead to a decrease in catches and yields? An example of two West African Coastal Lagoons. Fisheries and Management Ecologie 4 : 149-164. 1997.

Mohamed V. M. O. Etude de la dynamique des systèmes d'exploitation et de l'éco-biologie de la reproduction de trois Mugilidés: Mugil cephalus (Linnaeus, 1758), Liza aurata (Perguria, 1892) et Mugil capurrii (Risso, 1810), analyse de leurs stratégies d'occupations des secteurs littoraux mauritaniens et de leurs possibilités d'aménagement. Thèse de Doctorat de l’Université de Nice-Sophia Antipolis, France, 146 p. 2004.

Montchowui E., Laleye P., Philipart J.C., Poncin P. Biologie de la reproduction de Labeo parvus Boulanger, 1902 (Cypriniformes : Cyprinidae) dans le bassin du fleuve de l'oueme au Benin (Afrique de l ouest). Cahier d'éthologie, 22(2) : 61-802007.

N'Jock J.C. Les ressources démersales côtières du Cameroun : biologie et exploitation des principales espèces ichthyologiques. Thèse de doctorat de l’Université Aix Marseille 2, Tome I, 156 p. 1990.

Olapade J.O., Tarawallie S. The length-weight relationshipcondition factor and reproductive biology of pseudotolithus $(p)$ senegalensis (valenciennes, 1833) (croakers), in tombo western rural district of sierra leone. African Journal of Food, Agriculture, Nutrition and Development. 14 (6):93769389. 2014. 
Paugy D., Lévêque C., Teugels G. G. Faune des poissons d'eaux douces etsaumâtres de l'Afrique de l'Ouest. Tome II,IRD, Paris, 902 p1992.

Poulet N. Le sandre (Sander lucioperca L.) : Biologie, comportement etdynamique des populations en Camargue(Bouches de Rhône, France). Thèse deDoctorat de l’Université de Toulouse III,286 p. 2004.

Sidibé A. Liste rouge et conservation de la biodiversité marine dans la gestion écosystémique des pêches en Afrique de l'ouest. Programme marin et côtier pour l'Afrique centrale et occidentale, 58p.2010.

Sossoukpe E., Nunoo F. K. E., Dankwa H. R. Population structure and reproductive parameters of the Longneck croaker, Pseudotolithus typus (Pisces, Bleeker, 1863) in nearshore waters of Benin (West Africa) and their implications for management. Agricultural Science, 4 (6) : 9-18. 2013.

Sylla S., Atsé B.C., Kouassi N.J. Régime alimentaire de Trachinotus teraia (carangidae) dans la lagune Ebrié (cote d'ivoire), Cybium, 32 (1) : 81-87. 2009.

Varlet F. Le régime de la lagune Ebrié (Côte d’Ivoire). Traits physiques essentiels, Document ORSTOM, 83 : 164 p. 1978. 